\title{
Avaliação da Qualidade para Pesquisas em Educação em Engenharia de Software
}

\author{
Cleuton Almeida \\ Doutorado Profissional em Engenharia de \\ Software/CESAR SCHOOL \\ Rio Branco, Acre, Brasil \\ cma@cesar.school
}

As pesquisas em Educação em Engenharia de Software vêm crescendo nos últimos anos [3]. Essas discussões em torno deste contexto acontecem em eventos nacionais e internacionais e, portanto, há uma preocupação com a qualidade dessas pesquisas $[1,14]$. Entretanto, boa parte das pesquisas carecem de informações confiáveis quanto a validade dos resultados causados principalmente pela aplicação errônea ou equivocados dos métodos principalmente na pesquisa qualitativa envolvendo participantes humanos $[2,6,8,10,13]$ Isso pode se tornar um problema, pois, se pode estar acumulando hipóteses que se acreditam verdadeiras e que nunca foram verificadas empiricamente [7].

Muito embora exista uma quantidade substancial de recomendações metodológicas para garantir a qualidade de estudos em geral $[4,5,7,9,11,12]$, a falta de rigor na pesquisa em Educação em Engenharia de Software que envolvem participantes humanos permanece sob constantes críticas. Diante do problema apresentado fica evidente a necessidade de se buscar a qualificação das pesquisas em Educação em Engenharia de Software com participantes humanos. Em contraste com as diretrizes disponíveis em [4, 5, 7, 9, 11, 12], que se referem de forma mais geral à condução e relato de pesquisas genéricas, esta pesquisa de doutorado buscará identificar os elementos específicos da pesquisa em Educação em Engenharia de Software que envolvem participantes humanos.

Portanto, as questões de pesquisa que nortearão essa investigação serão: Que elementos determinam a qualidade da pesquisa em educação em SE com participantes humanos? Como avaliálos? E que aspectos devem ser observados pelos pesquisadores para garantir a qualidade das suas pesquisas, em tempo de planejamento?

Na primeira etapa da pesquisa será realizada uma Revisão Sistemática da Literatura que buscará responder a seguinte questão de pesquisa: Como se desenham as pesquisas de ensino em engenharia de software que envolvem participantes humanos nos principais fóruns nacionais dos últimos 10 anos? Quais são as principais limitações reportadas neste tipo de pesquisa

Na segunda etapa será realizada uma pesquisa qualitativa básica, com base em Entrevistas com pesquisadores de diferentes perfis - incluindo tanto pesquisadores da área de educação, quanto

Fica permitido ao(s) autor(es) ou a terceiros a reprodução ou distribuição, em parte ou no todo, do material extraído dessa obra, de forma verbatim, adaptada ou remixada, bem como a criação ou produção a partir do conteúdo dessa obra, para fins não comerciais, desde que sejam atribuídos os devidos créditos à criação original, sob os termos da licença CC BY-NC 4.0.

EduComp'21, Abril 26-30, 2021, Jataí, Goiás, Brasil (On-line)

(c) 2021 Copyright mantido pelo(s) autor(es). Direitos de publicação licenciados à Sociedade Brasileira de Computação (SBC)

\author{
César França \\ Departamento de Computação/Universidade Federal Rural \\ de Pernambuco(UFRPE) \\ Recife, Pernambuco, Brasil \\ cesar@franssa.com
}

da área de engenharia de software, e desde novatos até pesquisadores experientes que já participam amplamente de processos de avaliação de pesquisas -, que contribuem nos principais fóruns brasileiros de Educação em Engenharia de Software, sobre a percepção da qualidade destas pesquisas. Buscaremos avaliar, nesta etapa: Quais são os problemas e desafios encontrados em pesquisas de ensino em Engenharia de Software que envolvem participantes humanos? E como evitar estes problemas, ou transpor estes desafios?

A partir dos dados coletados nestas etapas iniciais, na terceira etapa desta pesquisa serão utilizados então técnicas específicas da Grounded Theory para combinar os dados do estudo literário com os dados advindos das entrevistas, a fim de sintetizar um conjunto de orientações guidelines.

Na quarta Etapa, propoe-se buscar uma avaliação e evolução deste conjunto de guidelines através da realização de Grupos Focais e Surveys novamente envolvendo diferentes perfis de pesquisadores, incluindo profissionais que atuam no ensino da engenharia de software mas que não necessariamente realizam pesquisas nesta área.

E, por fim, será utilizado um conjunto de Estudos de Caso para avaliar a contribuição prática do Guideline proposto.

\section{AGRADECIMENTOS}

Ao programa de Doutorado Profissional da CESAR SCHOOL por incentivar os estudantes a se tornarem verdadeiros pesquisadores. A meus pais: Nilce Nonata de Menezes e Vicente de Paula, por serem os responsáveis pelas minhas vitórias como pessoa, estudante e profissional. Aos meus filhos: Letícia, Daniel e Alice por serem a minha razão de viver. A minha esposa, Brunna Menezes, por me apoiar incondicionalmente em meus projetos.

\section{REFERÊNCIAS}

[1] ACM 2020. Association for Computing Machinery. Retrieved Set 11, 2020 from https://dl.acm.org/conferences

[2] Ahmed Al-Zubidy, Jeffrey C Carver, Sarah Heckman, and Mark Sherriff. 2016. A (updated) review of empiricism at the sigcse technical symposium. In Proceedings of the 47th ACM Technical Symposium on Computing Science Education. 120-125.

[3] José Adson OG da Cunha, Gabriel Araújo Marques, Wellington Lourenço Lemos, Uélio Dornelas Câmara Jr, and Francisco JS Vasconcellos. 2018. Software engineering education in Brazil: a mapping study. In Proceedings of the XXXII Brazilian Symposium on Software Engineering. 348-356.

[4] IEEE 2020. Review Criteria. Retrieved Oct 10, 2020 from https://ieeeedusociety. org/toe-scholarship-integration

[5] Devorah Kalekin-Fishman. 2001. David Silverman (2001). Interpreting Qualitative Data: Methods for Analysing Talk, Text and Interaction. In Forum Qualitative Sozialforschung/Forum: Qualitative Social Research, Vol. 2.

[6] Päivi Kinnunen, Veijo Meisalo, and Lauri Malmi. 2010. Have we missed something? Identifying missing types of research in computing education. In Proceedings of the Sixth international workshop on Computing Education Research. $13-22$. 
[7] Staffan Larsson. 2005. Om kvalitet i kvalitativa studier. Nordisk pedagogik 25, 1 (2005), 16-35

[8] Alex Lishinski, Jon Good, Phil Sands, and Aman Yadav. 2016. Methodological rigor and theoretical foundations of CS education research. In Proceedings of the 2016 ACM conference on international computing education research. 161-169.

[9] Austen Rainer and Sarah Beecham. 2008. Supplementary Guidelines, Assessment Scheme and evidence-based evaluations of the use of Evidence Based Software Engineering. University of Hertfordshire Technical Report, Version 2, 4 (2008).

[10] Justus J Randolph, George Julnes, Erkki Sutinen, and Steve Lehman. 2008. A methodological review of computer science education research. fournal of Information Technology Education: Research 7, 1 (2008), 135-162.
[11] Forrest Shull, Janice Singer, and Dag IK Sjøberg. 2007. Guide to advanced empirical software engineering. Springer.

[12] Sarah J Tracy. 2010. Qualitative quality: Eight "big-tent" criteria for excellent qualitative research. Qualitative inquiry 16, 10 (2010), 837-851.

[13] David W Valentine. 2004. CS educational research: a meta-analysis of SIGCSE technical symposium proceedings. ACM SIGCSE Bulletin 36, 1 (2004), 255-259.

[14] Avelino Francisco Zorzo, Daltro Nunes, Ecivaldo Matos, Igor Steinmacher, Renata Mendes de Araujo, Ronaldo Correia, and Simone Martins. 2017. Referenciais de Formação para os Cursos de Graduação em Computação. 